Reynerio M. Vencio, MD

Emmanuel Tadeus S. Cruz, MD

Department of Otorhinolaryngology

Head and Neck Surgery

Quezon City General Hospital
Correspondence: Reynerio M. Vencio, MD Department of Otorhinolaryngology-Head and Neck Surgery Quezon City General Hospital

Seminary Road, EDSA, Quezon City 1106

Philippines

Phone: 4261314 local 221

Email: reynerio_md@yahoo.com

Reprints will not be available from the author.

No funding support was received for this study. The authors signed a disclosure that they have no proprietary or financial interest with any organization that may have a direct interest in the subject matter of this manuscript, or in any product cited in this report.

Presented at the Surgical Instrumentation Contest (1 ${ }^{\text {st }}$ Place) Philippine Society of Otolaryngology Head \& Neck Surgery Annual Convention, Sofitel Philippine Plaza Manila

December 1, 2007

\section{An Improvised Tracheostoma Button Fabricated From Disposable Plastic Syringe}

\begin{abstract}
Objective: To design inexpensive, easily fabricated stoma buttons for post-laryngectomy patients with stomal stenosis and patients who require prolonged tracheotomy.
\end{abstract}

\section{Methods:}

Design: Surgical Instrumentation

Setting: Tertiary Government Hospital

Subjects: Tracheostoma buttons fabricated from 3 or 5 cc disposable plastic syringes were tested on two post-laryngectomy patients with stomal stenosis and a tracheotomized patient with bilateral vocal cord paralysis.

Results: The tracheostoma buttons were inexpensive and relatively easy to fabricate. They were easily inserted and well tolerated by all three patients, compared to previously-used commerciallyavailable tracheotomy tubes.

Conclusion: In a developing country setting, improvised tracheostoma buttons made from disposable plastic syringes may be viable alternatives to commercially-available stoma buttons or tracheotomy tubes. Clinical trials on more subjects should be conducted to assess parameters for use and long-term efficacy and safety issues.

Key words: stomal stenosis, tracheostoma/stoma button 
Tracheostomal stenosis (stomal stenosis) is a not uncommon complication following total laryngectomy. Its management varies with severity of respiratory compromise and different surgical techniques such as Single or Double ZPlasty and Lateral Skin Flap Technique (After Montgomery, 1963) ${ }^{1}$ have been advocated for correction, the main objective being to allow patients to breathe and function comfortably. The prospect of another surgical procedure causes undue psychological and financial stress and anxiety for many patients. But commercially-available post-laryngectomy products such as silicone laryngectomy tubes, stoma buttons or tracheostoma vents $^{2}$ which are alternatives to repeat surgery are not readily available in the local market and quite expensive.

In our setting, surgeons have resorted to long-term tracheotomy tubes to avoid stenosis but they have been observed to cause persistent cough due to chronic irritation of the tracheal wall.

We propose an improvised stoma button fabricated from disposable plastic syringes as inexpensive alternatives to prevent stomal stenosis for post-laryngectomy patients and for those who require prolonged tracheotomy for such conditions as tracheal stenosis and bilateral vocal cord paralysis.

\section{MATERIALS AND METHODS}

\section{Materials (Figure 1):}

1. 3 and 5 cc disposable plastic syringes (Terumo, Japan)

2. Cutter

3. Pen marker

4. Ruler

5. Cotton tip applicators

6. Compact 8 micromotor and hand drill (Soniford Maeller Corp., Phil.)

7. Leukoplast ${ }^{\mathrm{TM}}$ adhesive tape (BSN Medical, Malta)

8. Open flame candle or alcohol burner

9. Cloth tracheotomy tie or tiny gauze strips

\section{Procedure:}

1. With informed consent, the tracheostomal diameter of each patient was measured to determine the needed syringe size, 3 or $5 \mathrm{cc}$. The distance from the anterior edge of the stoma to the posterior tracheal wall was estimated with a cotton tip applicator (Figure 2) to determine the length of the stoma button minus half a centimeter to avoid contact with the posterior tracheal wall (Figure $3 a, b$ ).
2. The plunger was detached from the plastic syringe barrel and the distal end of the syringe barrel was cut diagonally at a $40^{\circ}$ angle with a cutter (Figure 4).

3. Holes for the attachment of strings were created on both sides of the neck plate of the barrel using a hand drill (Figure 5).

4. Sharp edges of the cut syringe were smoothened with flame (Figure 6).

5. The stoma button was now ready to use (Figure 7).

6. The appliance was inserted and with the aid of a penlight, the stomal edges and trachestoma button were examined for adequate dilation and retention, then secured with a cloth tracheotomy tie.

\section{RESULTS}

The improvised stoma buttons were used by two postlaryngectomy patients with stomal stenosis (Figure $8 a, b$ ) and one tracheotomized patient with bilateral cord paralysis (Figure 9). The buttons were relatively easy to fit and fabricate improving with the learning curve. Without need for stomal dilation, they were easily inserted in all three patients who all reported that the stoma button fit very well with less insertion pain and less foreign body sensation compared with their previous tracheotomy tubes. The patients also claimed to breathe better because of the wider diameter and absence of an indwelling cannula. The patients were followed up for four months with no noted complications.

\section{DISCUSSION}

Stomal stenosis is an often encountered problem following laryngectomy ${ }^{3}$. Short of performing surgical stomal revision or importing expensive, locally unavailable commercial stents, the improvised stoma button made from plastic syringe is a wise alternative.

The materials are readily available in the hospital setting and are inexpensive. Different plastic syringe sizes make it a versatile appliance to fit varying sizes of stoma and the procedure is easy to follow and can be duplicated.

Among the advantages of the improvised stoma button are: first, its length which is shorter than the usual tracheotomy tube resulting in less tracheal irritation; second, its lighter weight and smaller size make it more comfortable and acceptable to the patient; and third, its accessibility make it easier to clean when there are secretions.

Because it is shorter than a tracheotomy tube, the danger of 


\section{SURGICAL INNOVATIONS AND INSTRUMENTATION}

being dislodged during forceful coughing is greater. This can be minimized by tightening the button strings around the neck and by holding the button firmly against the stoma whenever coughing is anticipated. Matching the syringe size with stomal diameter to ensure a snug fit may also minimize accidental extrusion.

Complications from long-term use of the stoma button may include biofilm formation and stomal edge abrasions and granulation tissue. Periodic monitoring, daily cleaning and early intervention may prevent these complications. The shorter length of the stoma button and clearer plastic make such monitoring and cleaning easier. Clinical trials on more subjects should be conducted to assess parameters for use and long-term efficacy and safety issues including frequency of replacement. Improvements in design can evolve with experience.

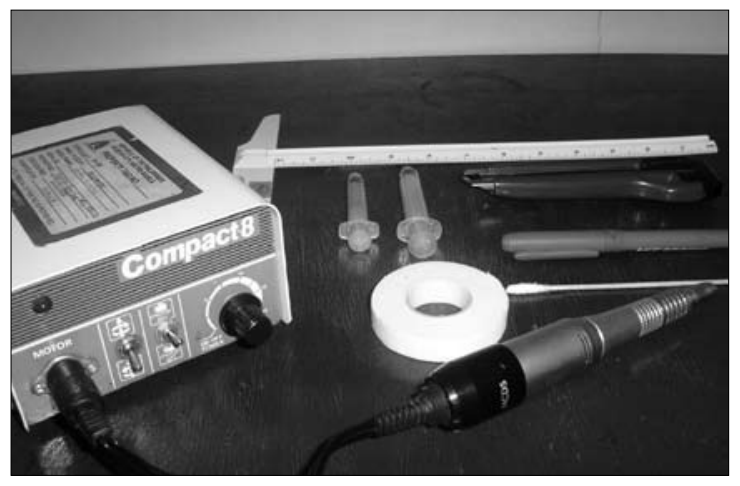

Figure 1. Materials

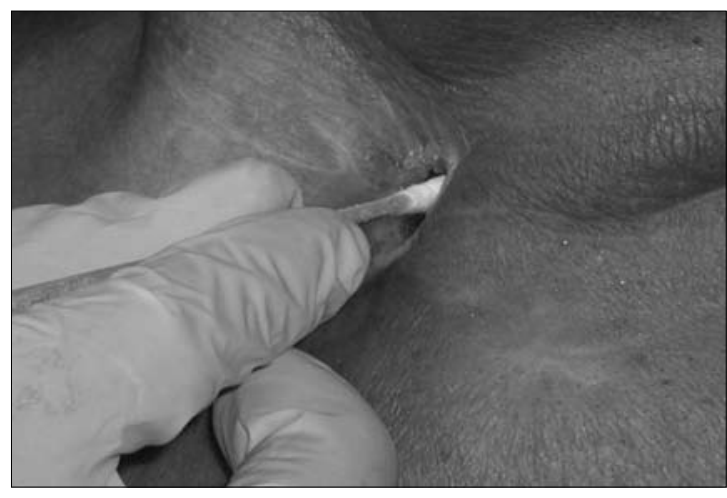

Figure 2. The distance from the anterior edge of the stoma to the posterior tracheal wall was measured with a cotton-tip applicator
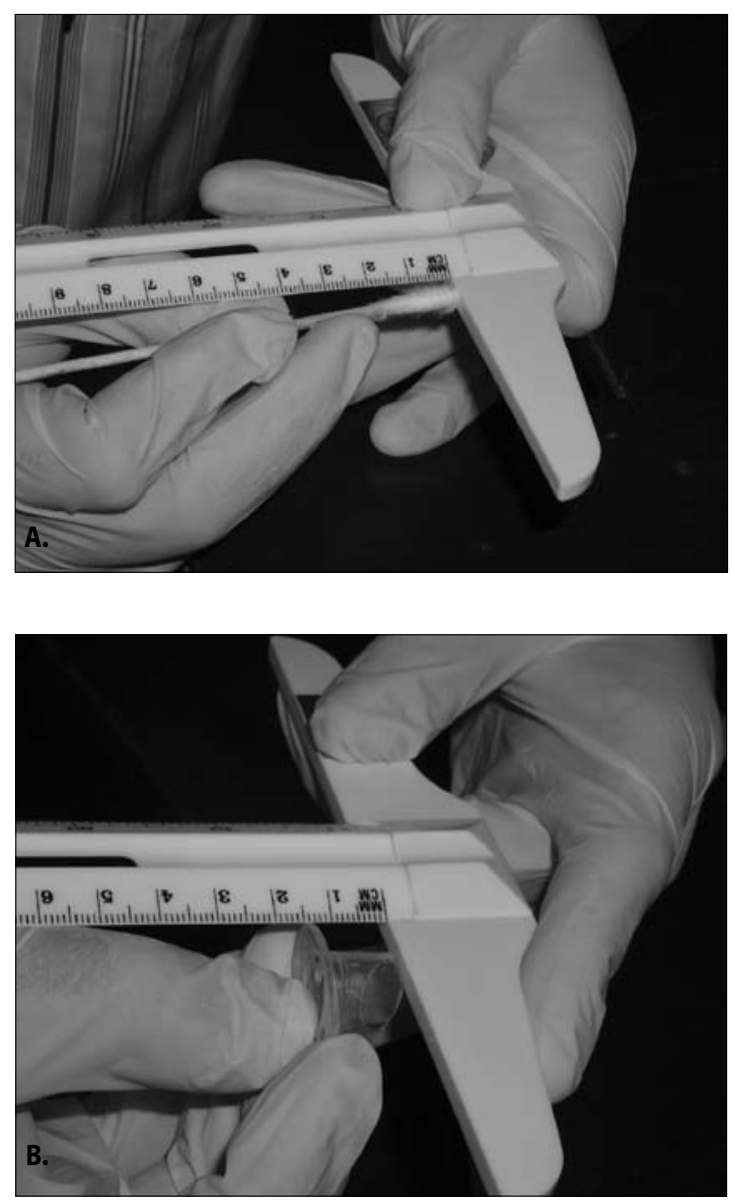

Figure $\mathbf{3 A}-\mathbf{B}$. The measurement determines the length of the stoma button minus half a centimeter to avoid contact with the posterior tracheal wall

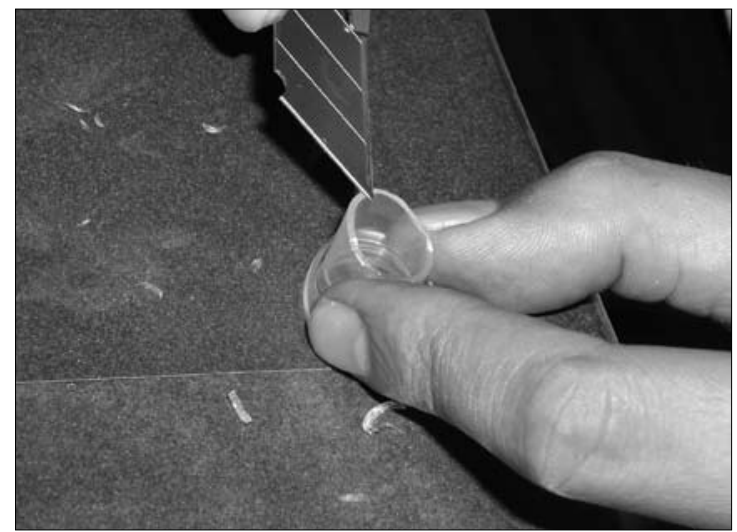

Figure 4. Diagonal cut made on the distal end of the barrel at a $40^{\circ}$ angle. 


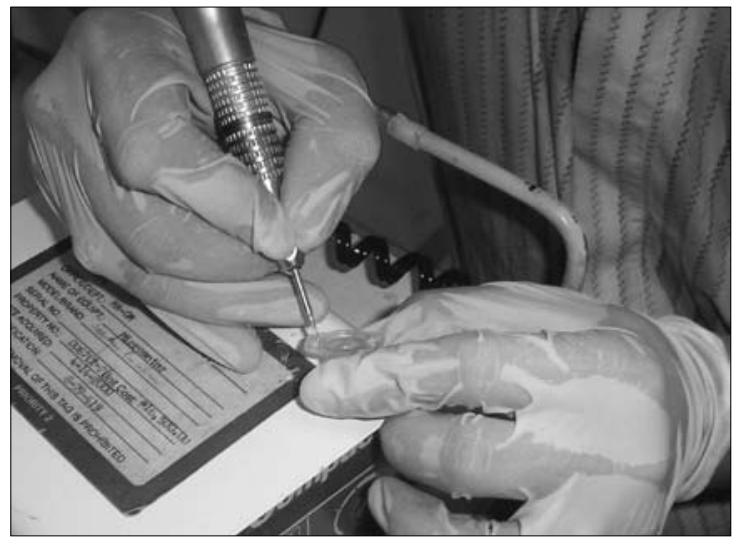

Figure 5. Holes for string attachment created on both sides of the neck plate of the barrel using a hand drill.

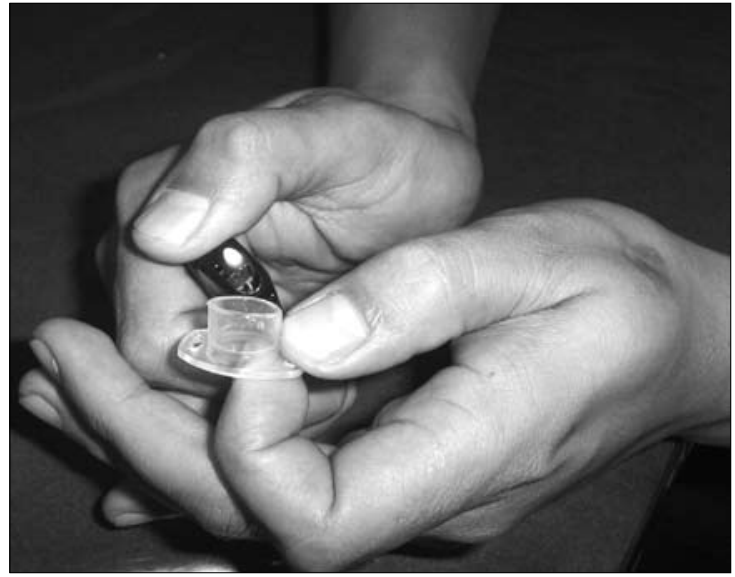

Figure 6. Sharp edges of the cut syringe smoothened with a flame.

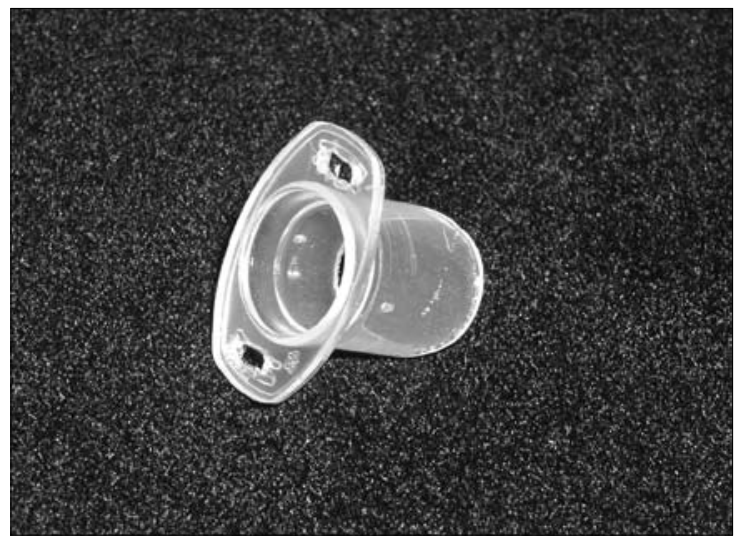

Figure 7. The improvised tracheostoma button.
Figure 8. Post-laryngectomy patients with stomal stenosis
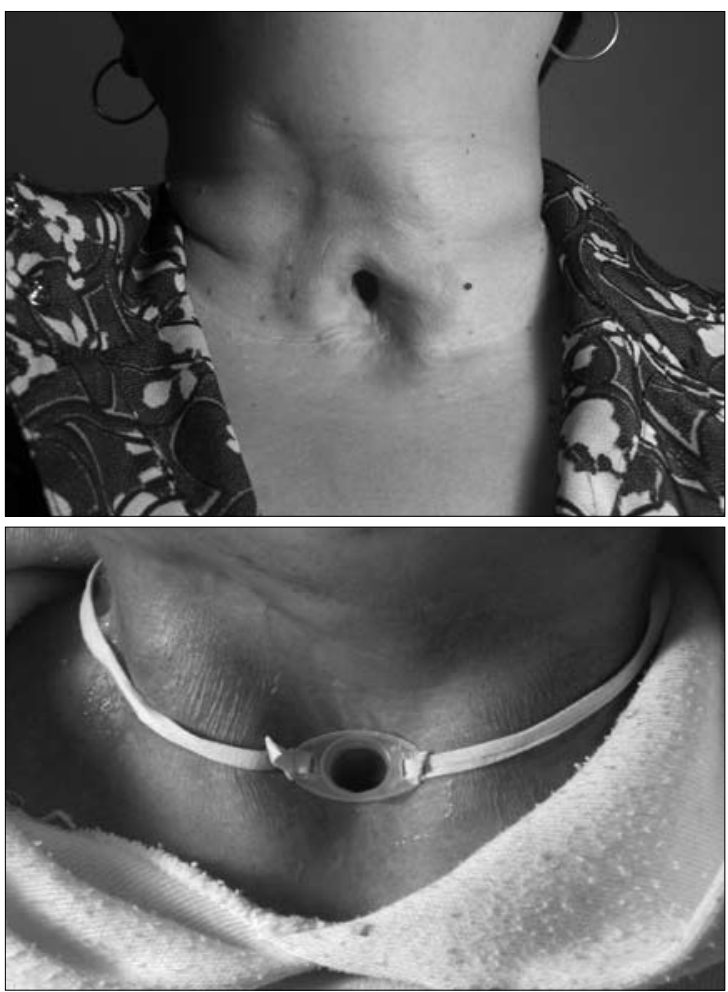

Figure $\mathbf{A}$.

72 year old female 7 months after total laryngectomy, pre (Top) and post (Above), button insertion
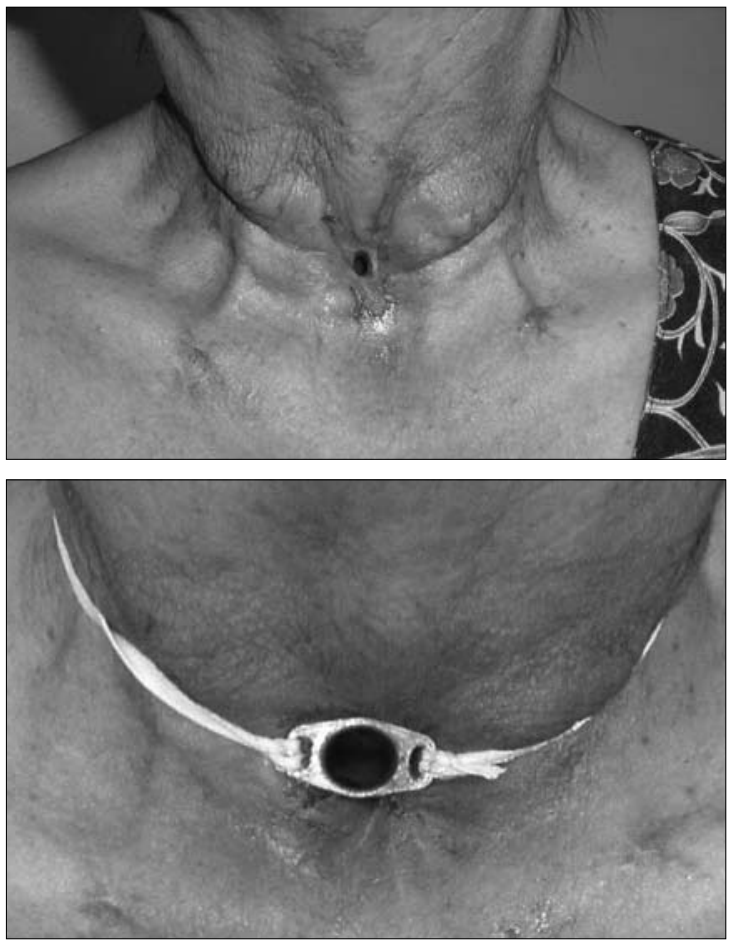

Figure $\mathbf{B}$.

65 year old female, 4 months after total laryngectomy, pre (Top) and post (Above), button insertion 
SURGICAL INNOVATIONS AND INSTRUMENTATION
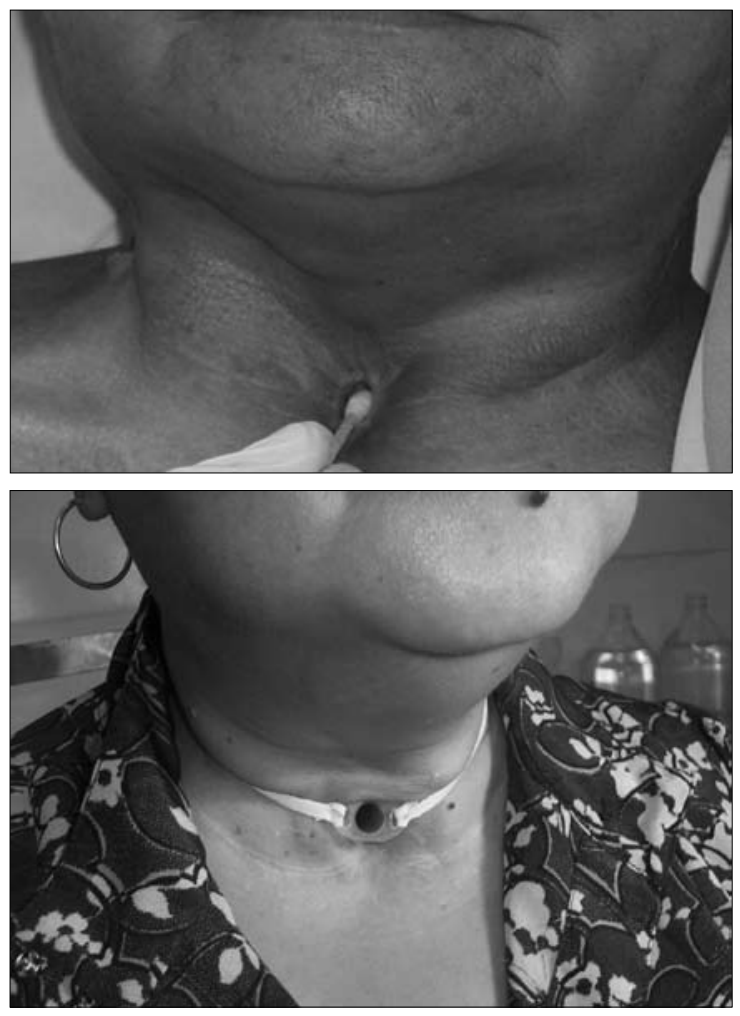

Figure 9.

Tracheotomized patient with bilateral vocal cord paralysis, pre (Top) and post (Above), button insertion

\section{REFERENCES}

1. Lore JM, Medina JE. An Atlas of Head and Neck Surgery. $4^{\text {th }}$ ed. Philadelphia: Elsevier Saunders; 2005

2. Messing BP, Hirata RM. (Kirklin Clinic Head and Neck Cancer Support Group Birmingham, AL). Tracheostomal Stenosis. Sanders PW, editor. Headlines: American Cancer Society; March 2005

3. Griffith GR, Luce EA. Tracheal Stomal Stenosis after Laryngectomy. Plast Reconstr Surg 1982; 70(6). 694-698 